\title{
On-sky performance demonstration of the near infrared SAPHIRA e-APD array and new developments of e-APD technology
}

\author{
Gert Finger *a, Ian Baker ${ }^{\mathrm{b}}$, Domingo Alvarez ${ }^{\mathrm{a}}$, Christophe Dupuy ${ }^{\mathrm{a}}$, Derek Ives ${ }^{\mathrm{a}}$, Leander Mehrgan ${ }^{\mathrm{a}}$, \\ Manfred Meyer ${ }^{\mathrm{a}}$, Jörg Stegmeier ${ }^{\mathrm{a}}$ and Harald J. Weller ${ }^{\mathrm{b}}$. \\ aEuropean Southern Observatory, Karl Schwarzschildstrasse 2, D-85748-Garching, Germany. \\ ${ }^{\mathrm{b}}$ Leonardo, Southampton, Hants, SO15 OLG, UK.
}

\begin{abstract}
A total of five near infrared SAPHIRA 320x256 pixel e-APD arrays have been deployed in the wavefront sensors and in the fringe tracker of the VLTI instrument GRAVITY. First results obtained by coherently combining the light of the four 8 meter telescopes of the VLTI have demonstrated diffraction limited performance with the CIAO adaptive optics system and a sensitivity improvement by a factor of 10 to 100 for interferometric observations. New APD developments since GRAVITY include the extension of the spectral sensitivity to the wavelength range from 0.8 um to $2.5 \mathrm{um}$. A larger format of 512x512 pixels is in development. At moderate APD gains the eAPD arrays have dark currents of $<2 \mathrm{E}-3$ e/s/pixel and outperform conventional state-of-the-art large format NIR science focal planes for integration times as long as $100 \mathrm{~s}$.
\end{abstract}

Keywords: avalanche photodiode, eAPD, $\mathrm{HgCdTe}$, readout noise, excess noise, APD gain, cryogenic amplifier, infrared, wavefront sensor, fringe tracker

\section{INTRODUCTION}

In 2007, ESO started a program at LEONARDO (former SELEX) to develop noiseless near infrared HgCdTe electron avalanche photodiode arrays (eAPD) [1][2]. These arrays were needed for the fringe tracker and the four wavefront sensors of the VLTI instrument GRAVITY to replace the initially baselined SPEEDSTER or PICNIC detectors. The eAPD technology is the only way to overcome the CMOS noise barrier of conventional near infrared high speed sensors used in control loops. The conventional sensors severely limit the performance of AO systems. Because of the noiseless APD gain inside the infrared pixel APD arrays are becoming the devices of choice for infrared AO wavefront sensors. After several development cycles of solid state engineering applied to the growth technology of metal organic vapour phase epitaxy (MOVPE), the eAPD arrays have matured and resulted in the SAPHIRA arrays. They have a format of $320 \times 256$ pixels with a pitch of $24 \mu \mathrm{m}$. They now offer an unmatched combination of subelectron read noise at $\mathrm{KHz}$ frame readout rates.

\section{ARCHITECTURE OF SAPHIRA ROIC}

ESO funded a new custom specific ROIC design tailored to the special needs of the GRAVITY fringe sensor and the VLTI wavefront sensors. To keep the cost low, the same format as used in a previous ROIC was used with $320 \times 256$ pixels and a pixel pitch of $24 \mu \mathrm{m}$. The AO control loops require frame rates of $1 \mathrm{kHz}$. Therefore, by providing 32 parallel video outputs, the required analog bandwidth and the associated readout noise can be reduced to $5 \mathrm{Mpixel} /$ output.

*gfinger@eso.org; phone +49-8161-234738; www.eso.org/ gfinger, www.eso.org 
GRAVITY does not need to read the full frame. The 48 spectrally dispersed fringes of the fringe tracker can be read by 24 separate windows of $32 \times 3$ pixels. The 68 active subpupils of the CIAO wavefront sensor sampled by $8 \times 8$ pixels require a window size of $96 \times 72$ pixels. To optimize the windowed readout, the 32 outputs are organized in such a way that they read out 32 adjacent pixels in a row at the same time. Hence, the windowed readout also benefits from the multiplex advantage of 32 parallel channels. However, the window size must be a multiple of 32 in the row direction, whereas there are no size restrictions in the column direction. The regions of interest and the regions which are reset can be separately downloaded in advance through a serial programming interface. The unit cell of the ROIC is a state of the art low noise source follower. The array operates in the capacitive discharge mode, which allows multiple nondestructive readouts and Fowler sampling. Double correlated readout in the rolling shutter mode with read-reset-read by row and a duty cycle of $100 \%$ at the maximum frame rate is also possible. The time to read out a full frame is $512 \mu$ s scaling with the window size for smaller windows. The layout of the SAPHIRA ROIC is shown in Figure 1. Regions of interest (green) and reset regions (yellow) can be predefined allowing different integration times in different windows.

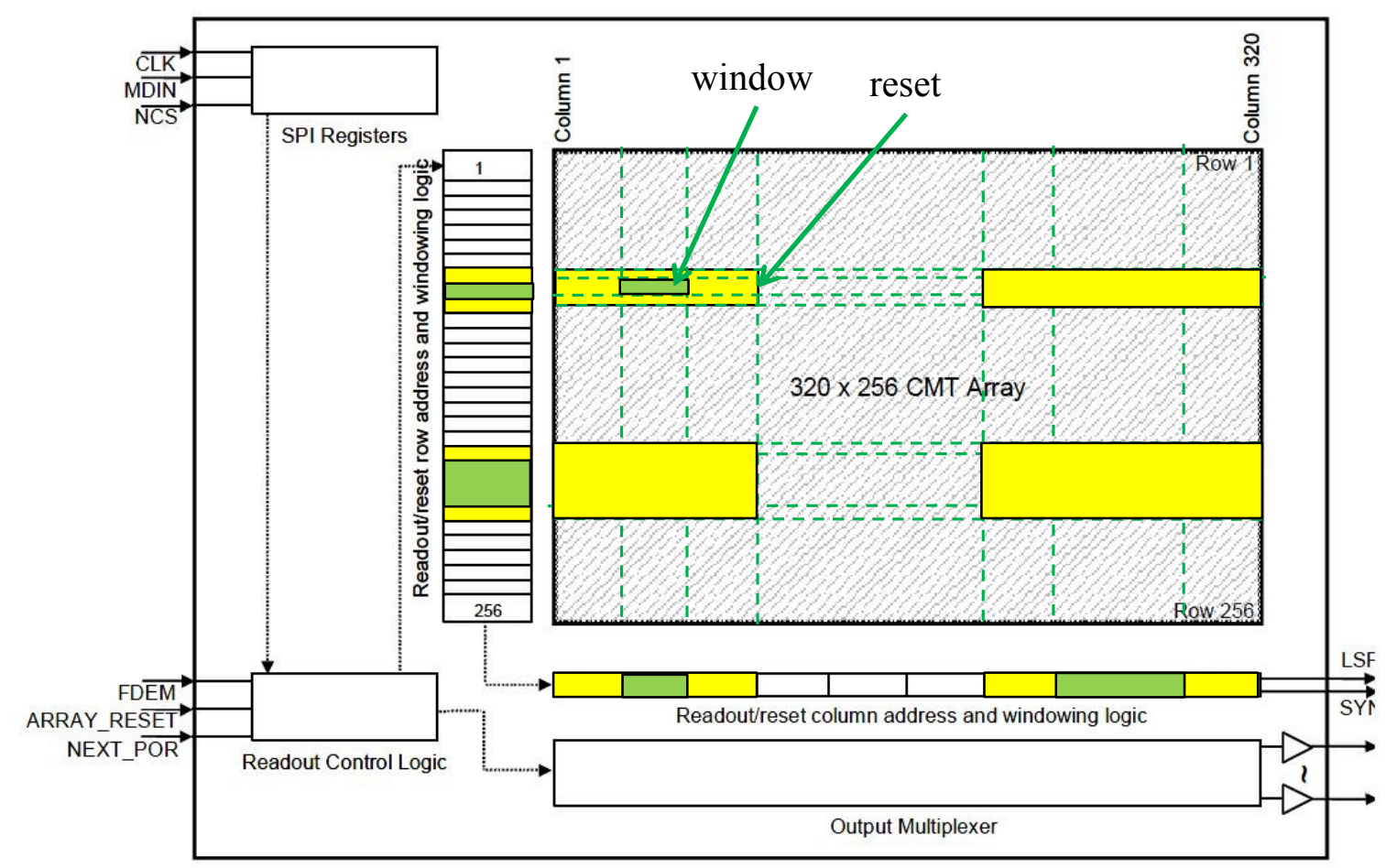

Figure 1 Layout of the SAPHIRA ROIC with predefined regions of interest (green) and reset regions (yellow).

\section{DESIGN OF MOVPE GROWN DIODE}

The first $\lambda \mathrm{c}=2.5 \mu \mathrm{m}$ HgCdTe arrays hybridized to the SAPHIRA ROIC were Mark3 diode designs shown on the left side of Figure 2. The arrays are mesa heterojunctions grown by MOVPE on a cheap GaAs substrate which is then removed by a chemical etch. The top layer is a CdTe seed layer which is opaque at wavelengths $\lambda<0.8 \mu \mathrm{m}$. A thick wide bandgap buffer layer is used to cope with the lattice mismatch of GaAs. This buffer layer limits the spectral response of Mark3 on the short wavelength side to wavelengths $\lambda>1.3 \mu \mathrm{m}$. A mesa slot extending through the absorber layer electrically isolates the pixels. Photons are absorbed in the p-type absorber. The photon generated charge diffuses to the $p-n$ junction and is then accelerated in the electric field of the gain region to start the multiplication process by impact ionization. To 
boost the avalanche gain, the multiplication region is made of narrow bandgap material corresponding to a cutoff wavelength of $\lambda_{c}=3.5 \mu \mathrm{m}$. The Mark3 diode arrays are currently deployed in the GRAVITY instrument at the VLTI.

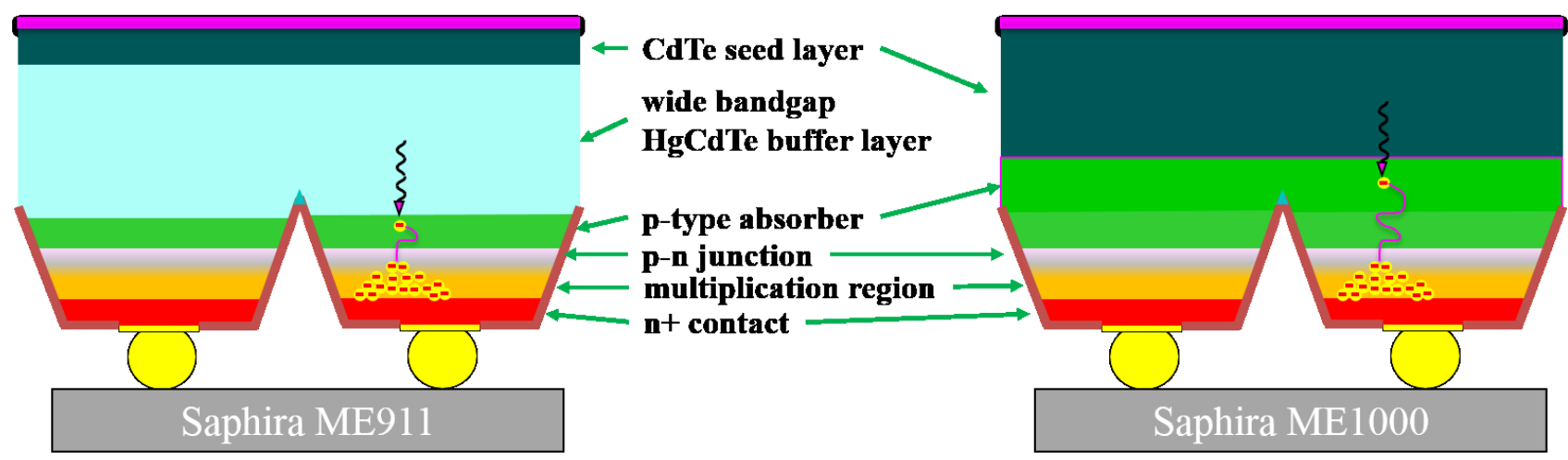

Figure 2 Left: Mark3 MOVPE diode design with wide bandgap buffer layer limiting short wavelength response to $\lambda>1.3 \mu \mathrm{m}$. Right: Mark14 MOVPE diode design without wide bandgap buffer layer sensitive from 0.8 to $2.5 \mu \mathrm{m}$

To include the astronomically important $\mathrm{Y}$ and $\mathrm{J}$ bands in the spectral range of eAPDs, the Mark14 diode structure was developed. This development was funded by the University of Hawaii [5][6]. To extend the spectral response down to $\lambda=0.8 \mu \mathrm{m}$ the wide bandgap buffer layer had to be removed. The absorber layer is directly grown on CdTe as shown on the right side of Figure 2. The p-type absorber is now much thicker and therefore requires much longer diffusion lengths. This is achieved by a high temperature anneal.

\section{PERFORMANCE OF SAPHIRA ARRAY}

$\mathrm{HgCdTe}$ is a direct semiconductor and thus an ideal material for electron avalanche photodiodes. Since the mass of the electron is much smaller than the mass of the hole only electrons are multiplied by an almost noiseless APD gain process. Depending on the temperature we measured an excess noise factor between 1.1 and 1.3 at an APD gain of 400 . Apart from the extended wavelength range the removal of the wide bandgap buffer layer - realized for the first time in Mark14 arrays - resulted in high APD gain with superb cosmetic quality, which is maintained up to the highest APD gain as shown in Figure 3.

The blue diamonds in the plot below show that the APD gain measured in H-band at an operating temperature of $60 \mathrm{~K}$ is as high as 637 for a bias voltage of $19.1 \mathrm{~V}$. The APD gain in K-band is almost as high as in H-band as shown by the red squares. This demonstrates that the thickness of the absorber is sufficient for the K-band photons to be absorbed in the absorber layer. As a consequence, photon generated electrons experience the full APD gain, which has not been the case in some of the previous diode designs. The flatfield taken in K-band, which is inserted in the plot, demonstrates the cosmetic quality of the array. Even at an APD gain as high as 126, the array has $99.95 \%$ operability or only 40 bad pixels. An operable pixel is defined here as a pixel which has a signal deviating by less than $30 \%$ from the mean signal. we measured an excess noise factor between 1.1 and 1.3 at an APD gain of 400. Apart from the extended wavelength range the removal of the wide bandgap buffer layer - realized for the first time in Mark14 arrays - resulted in high APD gain with superb cosmetic quality, which is maintained up to the highest APD gain. The blue diamonds in the plot below show that the APD gain measured in H-band at an operating temperature of $60 \mathrm{~K}$ is as high as 637 for a bias voltage of 19.1V. The APD gain in K-band is almost as high as in H-band as shown by the red squares. This demonstrates that the thickness of the absorber is sufficient for the K-band photons to be absorbed in the absorber layer. As a consequence, photon generated electrons experience the full APD gain, which has not been the case in some of the previous diode designs. The flatfield taken in K-band, which is inserted in the plot, demonstrates the cosmetic quality of the array. Even at an APD gain as high as 126 , the array has $99.95 \%$ operability or only 40 bad pixels. An operable pixel is defined here as a pixel which has a signal deviating by less than $30 \%$ from the mean signal. Since the SAPHIRA arrays are used in the window mode as wavefront sensors in the Coude Infrared Adaptive Optics (CIAO) systems of the four 8 meter VLT telescopes the effect of multiple sampling on the noise of this readout mode was investigated in more detail. The CIAO system employs a Shack-Hartmann lenslet array with 9x9 subapertures. Each subaperture has 8x8 pixels. Therefore, an 


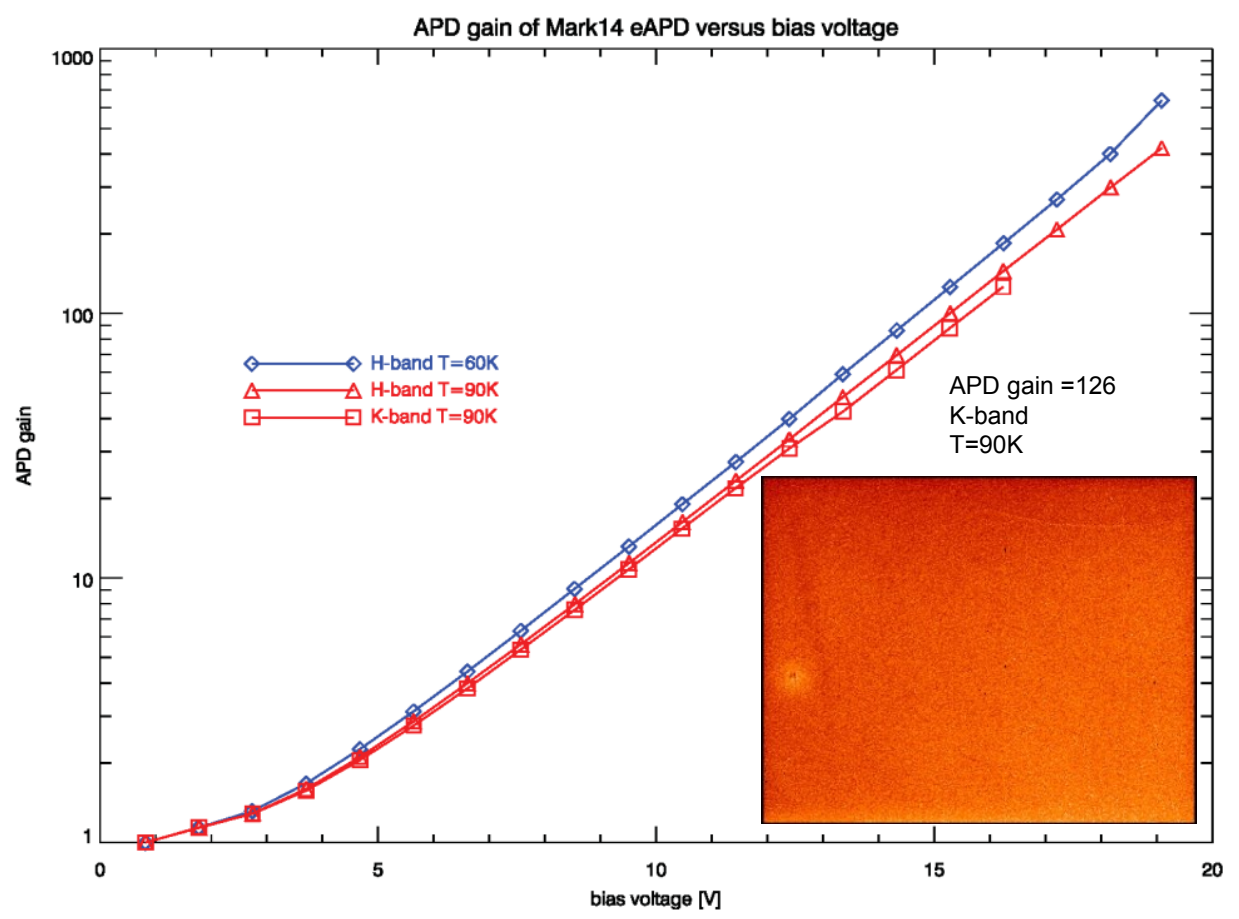

Figure 3 APD gain as a function of bias voltage. Inserted image is flatfield at APD gain of 126 taken in K-band at a temperature of $\mathrm{T}=90 \mathrm{~K}$ showing superb cosmetic quality at high APD gain.

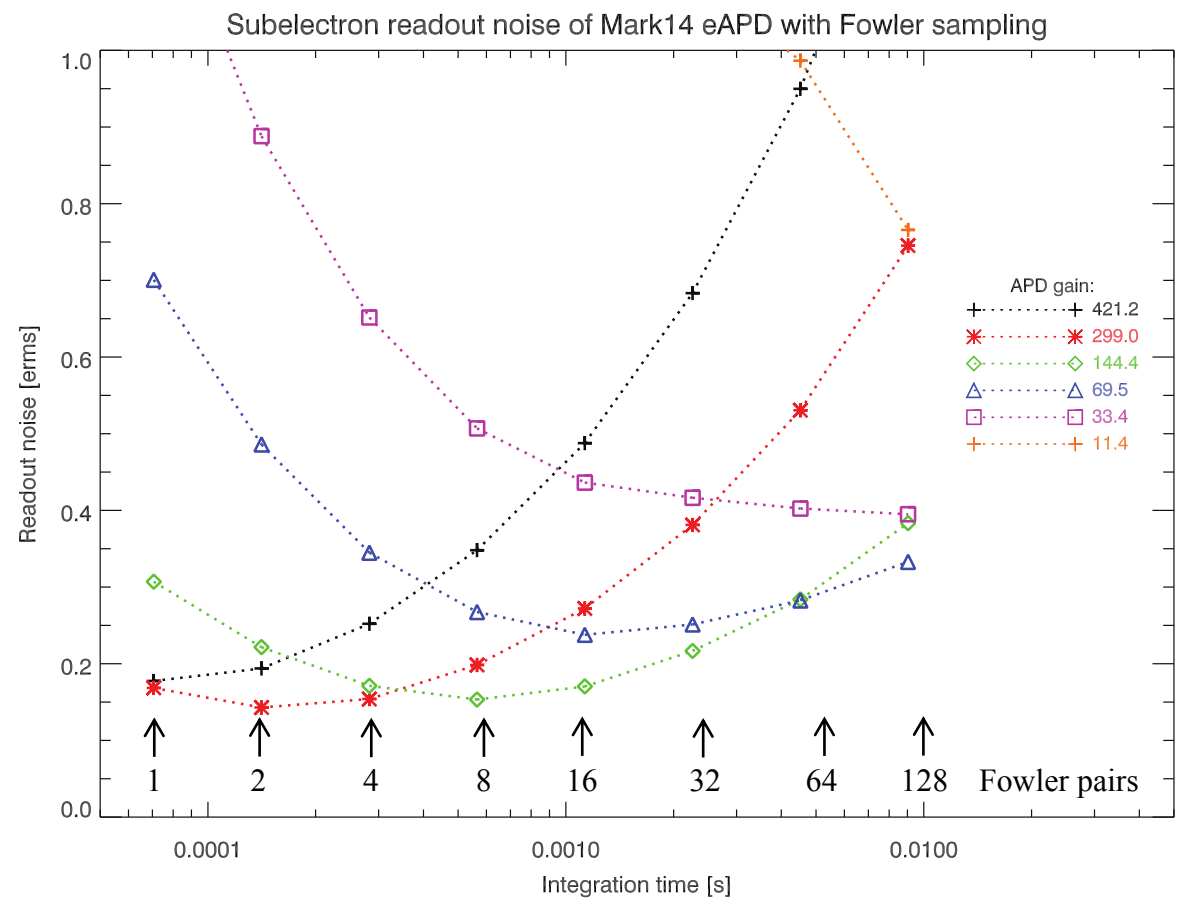

Figure 4 Subelectron readout noise of Mark14 eAPD at a detector temperature of T=90K for different APD gains. Number of Fowler pairs is proportional to detector integration time and increase by a factor of 2 for each data point. Number of Fowler pairs from left to right: 2,4,8,16,32,64 and 128 . 
area of $72 \times 72$ pixels is needed. Because of the special readout topology of the SAPHIRA ROIC with 32 channels reading out 32 adjacent pixels simultaneously, the smallest window covering this area is a 96x72 pixel window. For this window size, the measured readout noise versus the integration time is plotted in Figure 4 measured at different APD gains.

The range of the vertical axis is zero to one electron rms. Therefore, the noise of all measured points is subelectron. The detector integration time in the plot is proportional to the number of Fowler pairs and increases by a factor of 2 for each data point. The number of Fowler pairs increases from left to right from 1 to 2,4,8,16,32,64 and 128 Fowler pairs. As shown by Figure 5 the quantum efficiency $\mathrm{QE}(\lambda)$ is high over the entire sensitive range from $\lambda=0.8 \mu \mathrm{m}$ to $2.5 \mu \mathrm{m}$. It was measured by scaling the relative quantum efficiency to the absolute values measured in the $\mathrm{H}$ and $\mathrm{K}$ bands. At unity APD gain, represented by the solid line, the array is sensitive from $\lambda=0.8 \mu \mathrm{m}$ to $\lambda=3.5 \mu \mathrm{m}$ due to the narrow bandgap gain layer. For an APD gain of 221 the quantum efficiency is represented by the dash dotted curve. Only those electrons which have been generated by photons with a wavelength shorter than the cutoff wavelength of the absorber layer experience the full APD gain. The spectral quantum efficiency at short wavelengths shows a modulation characteristic of interference fringes generated by multiple reflections in the CdTe layer.

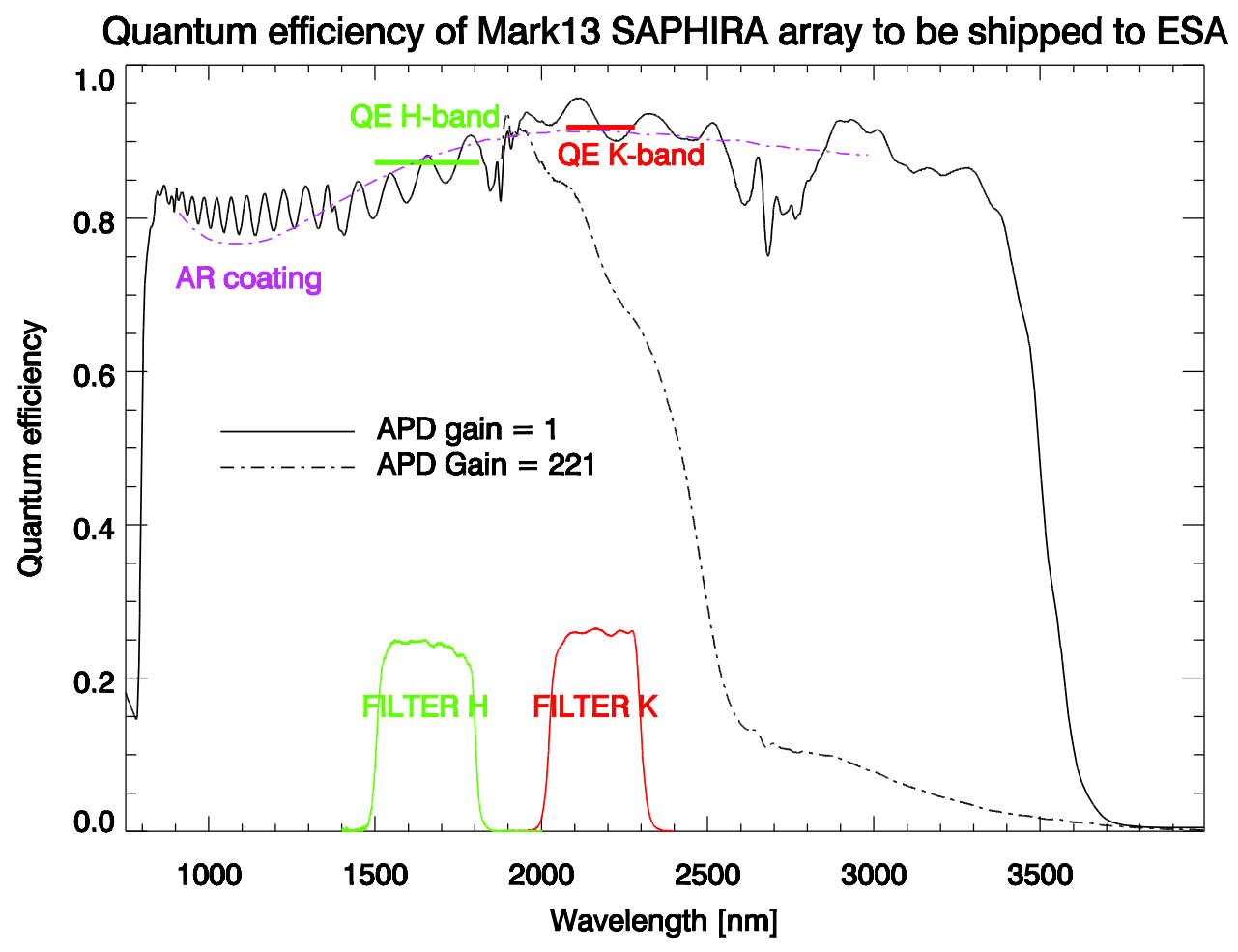

Figure 5 Quantum efficiency of Mark13 array versus wavelength for unity APD gain (solid curve) and APD gain of 221(dash-dotted curve). At unity APD gain cutoff wavelength is $3.5 \mu \mathrm{m}$ due to narrow bandgap gain region. At high APD gain cutoff wavelength is $2.5 \mu \mathrm{m}$ since photons with wavelengths longer than the cutoff wavelength of the absorber layer experience only partial APD gain. Absolute quantum efficiency is based on measurements with broad band $H$ and $K$ filters as indicated in plot. $Q E$

\section{ON-SKY PERFORMANCE OF SAPHIRA IN GRAVITY}

Both the fringe tracker and the wavefront sensors outperform the specifications of GRAVITY [3][4]. The Coude Infrared Adaptive Optics systems are located in the Coude rooms of the VLT telescopes and have bimorph deformable mirrors. 
The Shack-Hartmann wavefront sensors have 9x9 subapertures with a FOV of 2" sampled by 4x4 pixels and separated by 8 pixels. Figure 6 shows the closed loop rejection transfer function for Zernike modes up to order 44 . The Strehl ratios as a function of seeing measured on a $\mathrm{mK}=6.5 \mathrm{mag}$ star is shown in Figure 7 . The inserted images were taken with the AO loop on and off. For good seeing CIAO works on guide stars as faint as $11 \mathrm{mag}$.

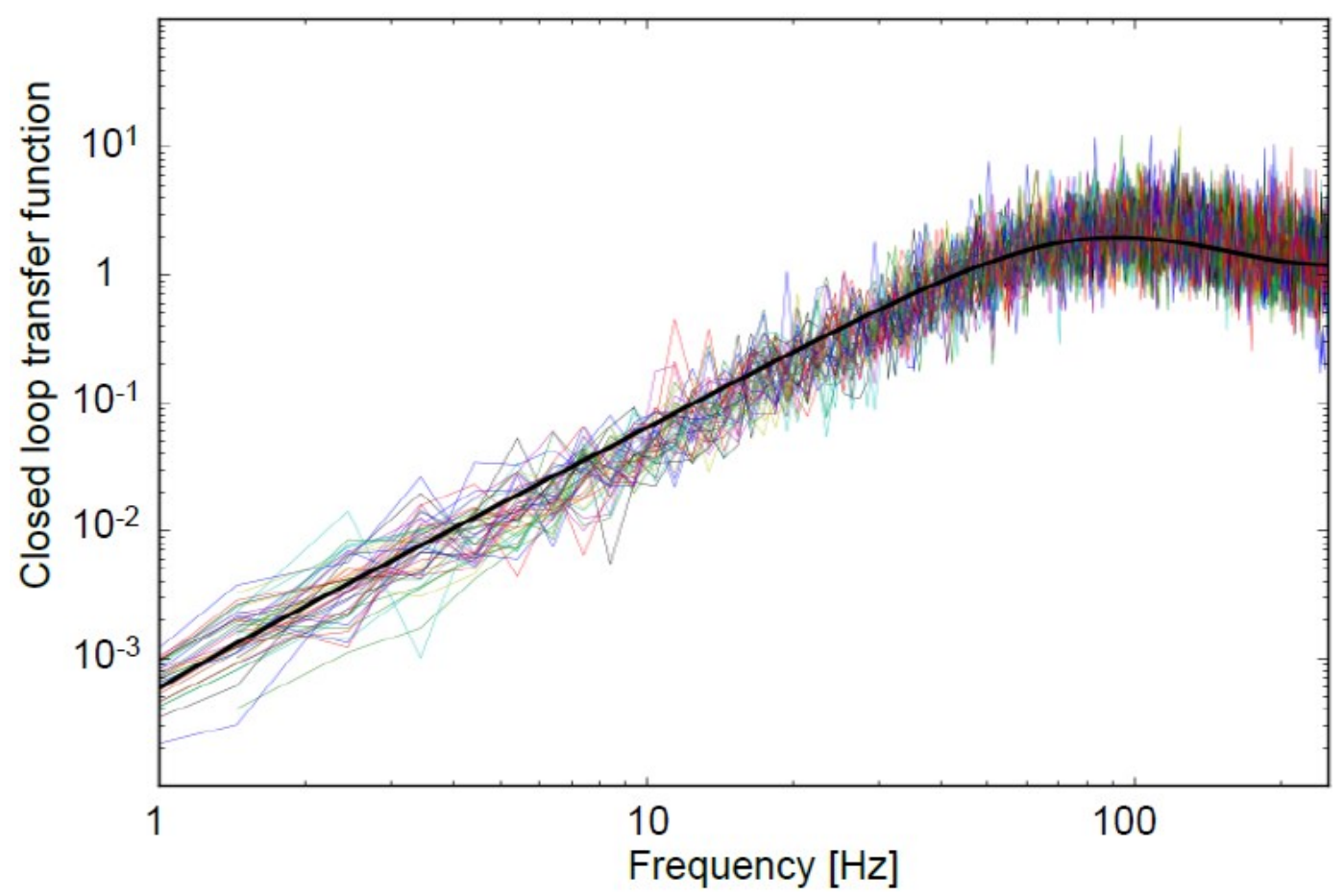

Figure 6 Coude Infrared Adaptive Optics (CIAO) closed loop rejection transfer function for Zernike modes up to order 44.

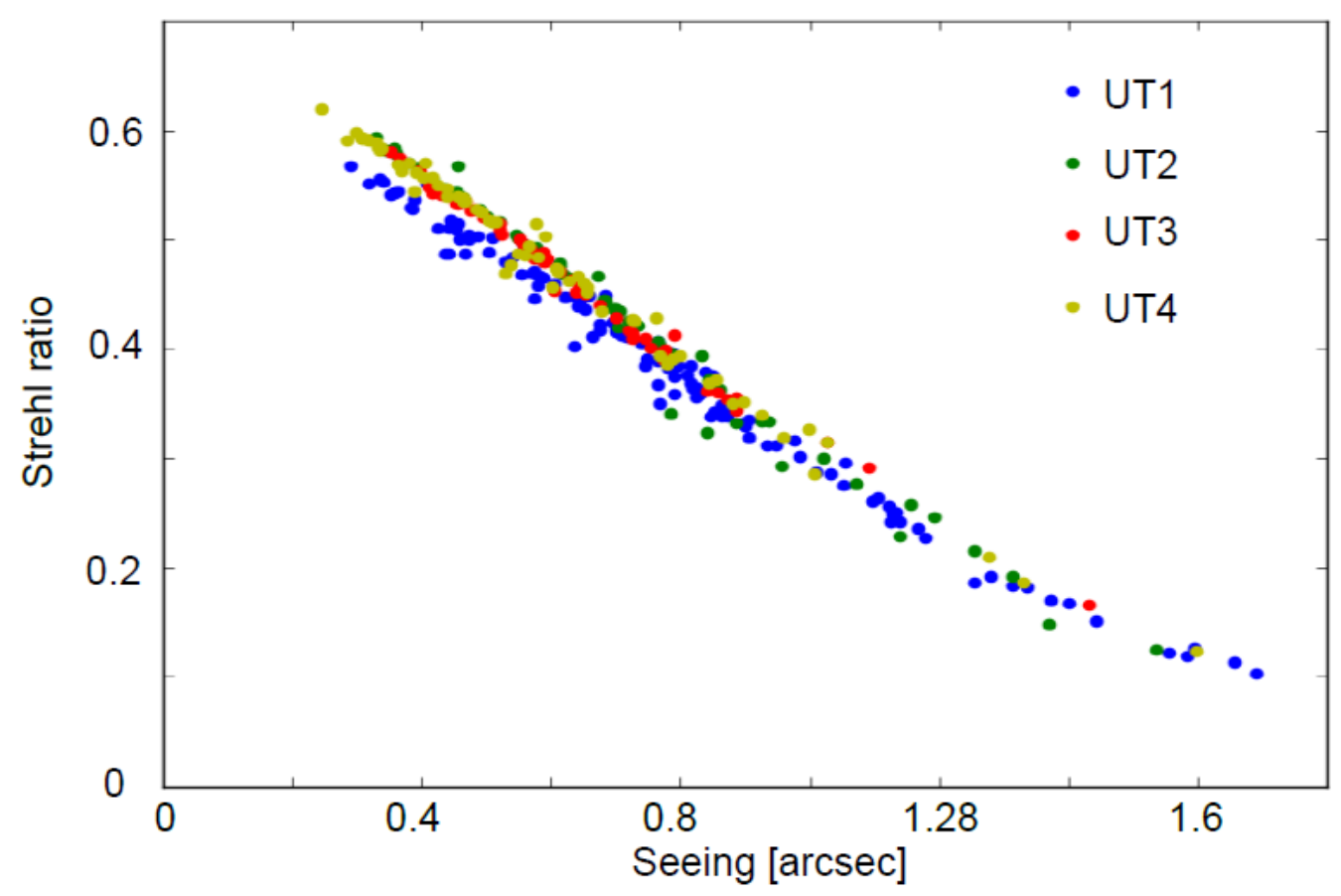

Figure 7 Strehl ratio of CIAO as a function of seeing for a star of magnitude 6.5. 
On the left side of Figure 8, fringes of IRS16C $(\mathrm{mK}=10)$ are shown which are taken with the SAPHIRA fringe tracker to stabilize the dispersed science fringes (middle) of the star S2 $(\mathrm{mK}=14)$ in the Galactic center, shown on the right side. The limiting magnitude for coherent exposures with GRAVITY is $\mathrm{mK} \sim 17$ - this sets a new sensitivity standard in infrared interferometry, made possible by the deployment of eAPD technology.

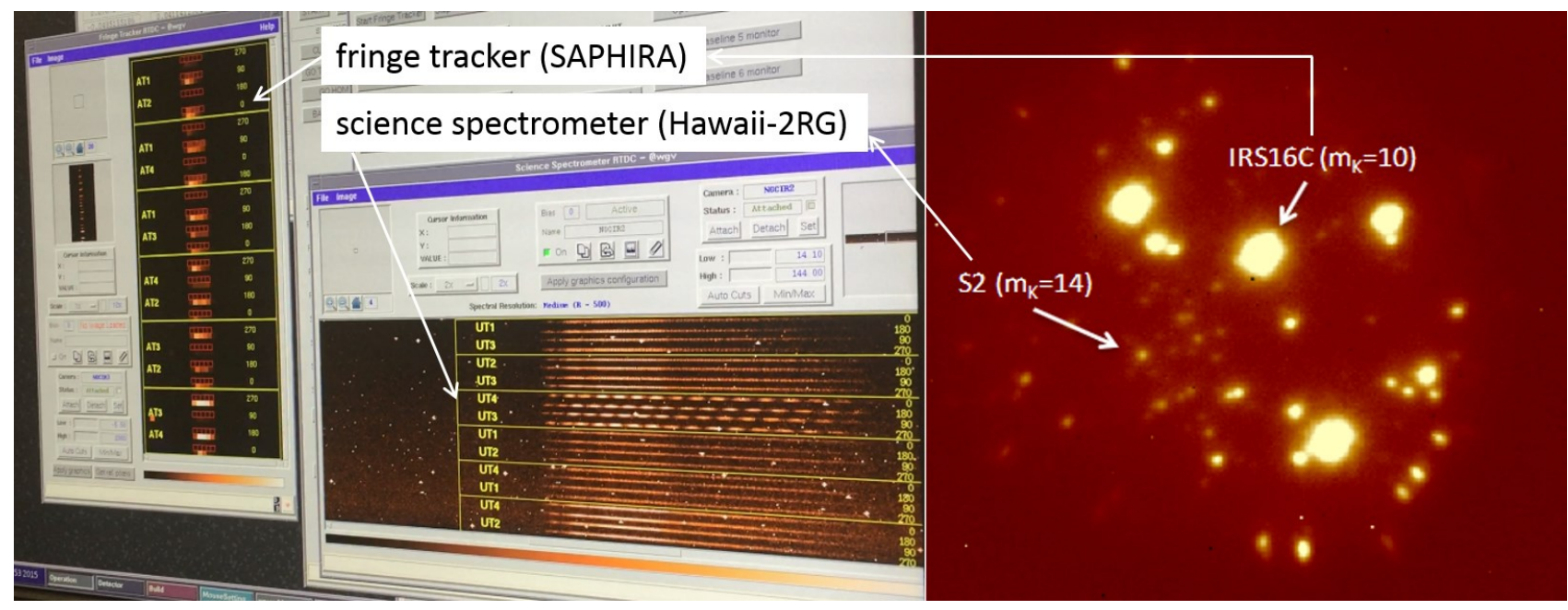

Figure 8 Middle: Dispersed and stabilized fringes of star $S 2\left(m_{K}=14\right)$ measured with science spectrometer. Right: Image of galactic center with S2 and IRS16C $\left(\mathrm{m}_{\mathrm{K}}=14\right)$, which is used for fringe tracking. Right: Dispersed spectra of IRS16C on SAPHIRA fringe tracker.

\section{LARGE FORMAT SAPHIRA}

Recently, major funding was made available to the University of Hawaii by the Astrophysics Research and Analysis Program APRA of NASA's Science Mission Directorate for the development of a $1 \mathrm{~K} \times 1 \mathrm{~K}$ class photon counting $\mathrm{HgCdTe}$ eAPD array at LEONARDO optimized for low background space astronomy [5][6]. The pixel size for this large format has to be downsized from $24 \mu \mathrm{m}$ to $15 \mu \mathrm{m}$ or $12 \mu \mathrm{m}$ which involves a certain risk.

Apart from the small format the current SAPHIRA array meets all specifications of the ELT near infrared wavefront sensors. Therefore, ESO together with MPE and NRC Herzberg decided to fund a complementary development of a larger 512x512 pixel high speed AO optimized eAPD array. The layout of the large SAPHIRA array is shown in Figure 9. The larger formats is needed for the ELT wavefront sensors of the pre-focal station, for the sensor to co-phase the ELT mirror segments, for the pyramid wavefront sensor of the second generation ELT Planetary Camera and Spectrograph PCS and for the WFS of the mid-infrared imager and spectrometer METIS. To keep both risk and cost low, a fast approach was chosen to develop a $512 \times 512$ pixel SAPHIRA array with the leaving the proven technological elements of the current SPAHIRA array unchanged as far as possible. The pixel size of $24 \mu \mathrm{m}$ will be maintained since this size is favored by the instrument consortia. The CMOS ROIC will remain a $0.6 \mu \mathrm{m} 5 \mathrm{Vdesign}$. The readout architecture will be similar to the current SAPHIRA array with the same multiplex advantage for windowed readout but split into two halves symmetric to the central row. Each half has 32 video outputs and is read form the top or bottom edge towards the center. The video outputs will be read with $8.7 \mathrm{Mpixel} / \mathrm{channel}$. The frame rate for the full array will be $1 \mathrm{Kfps}$ for DCS and $2 \mathrm{Kfps}$ for uncorrelated readout. Special care will be taken to provide glow protection which will allow to also use this ROIC to develop diode structures for low flux science focal planes. 


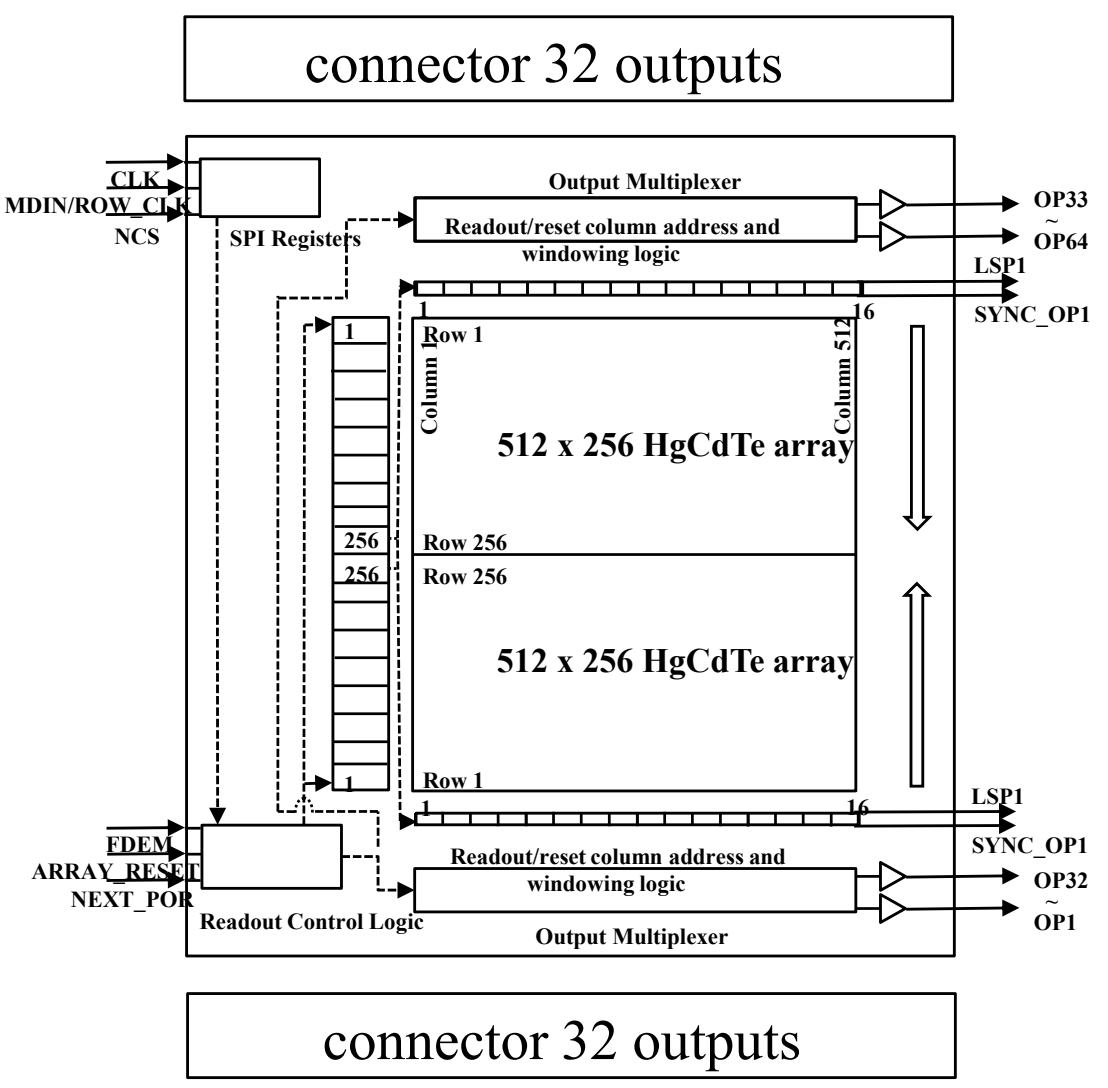

Figure 9 Large Saphira array with format of $512 \times 512$ pixel. ROIC is split in two halves with 32 outputs at bottom and 32 outputs at top. Pixel rate per output is $8.7 \mathrm{MHz}$. Frame time is $2 \mathrm{Kframes} / \mathrm{s}$ for uncorrelated sampling.

\section{EAPD TECHNOLOGY FOR SCIENCE FOCAL PLANES?}

At low APD gain the dark current of the current Mark 14 SAPHIRA array is $<1 \mathrm{E}-3$ electron/s/pixel which is as low as the dark current of the best MBE grown HgCdTe arrays. The dark current histogram is shown in Figure 10. At a moderate APD gain of 7.7 and for an integration time of 100s the readout noise of the SAPHIRA Mark14 array outperforms state-of-the-art conventional $2 \mathrm{Kx} 2 \mathrm{~K}$ CMOS arrays as demonstrated by the noise histograms of a SAPHIRA array and a large format conventional $2 \mathrm{Kx} 2 \mathrm{~K} \mathrm{HgCdTe} \mathrm{CMOS}$ array in Figure 11 . For a fair noise comparison, the ratio of the readout times is equal to the ratio of the formats. Bothe arrays have been channel-offset corrected. An optimized diode structure is in development with a wider bandgap in the gain region to increase the APD gain without the onset of trap assisted tunneling dark current. This will result in better sensitivity. 


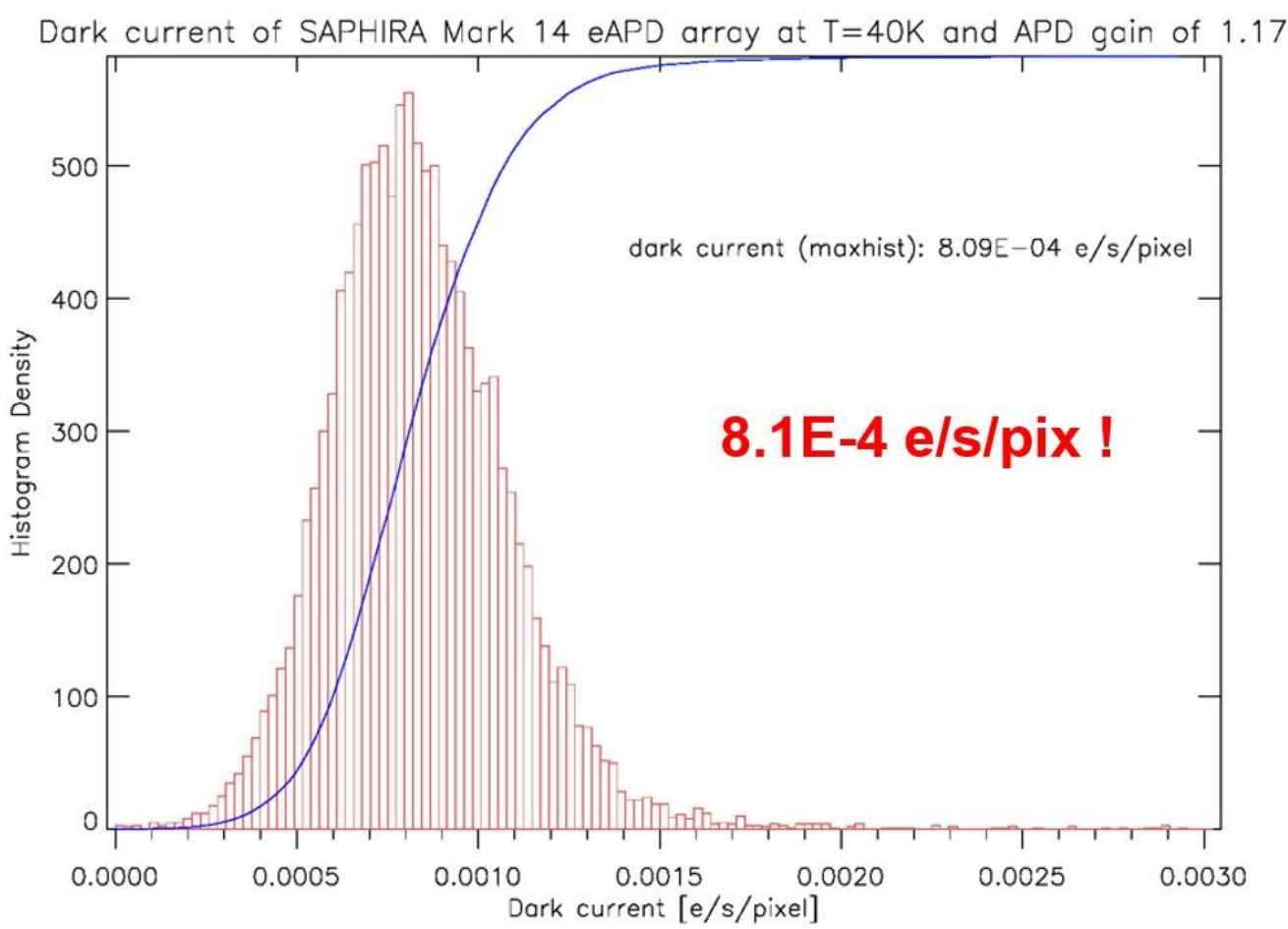

Figure 10 Dark current histogram of SAPHIRA array at $\mathrm{T}=\mathbf{3 8 K}$.

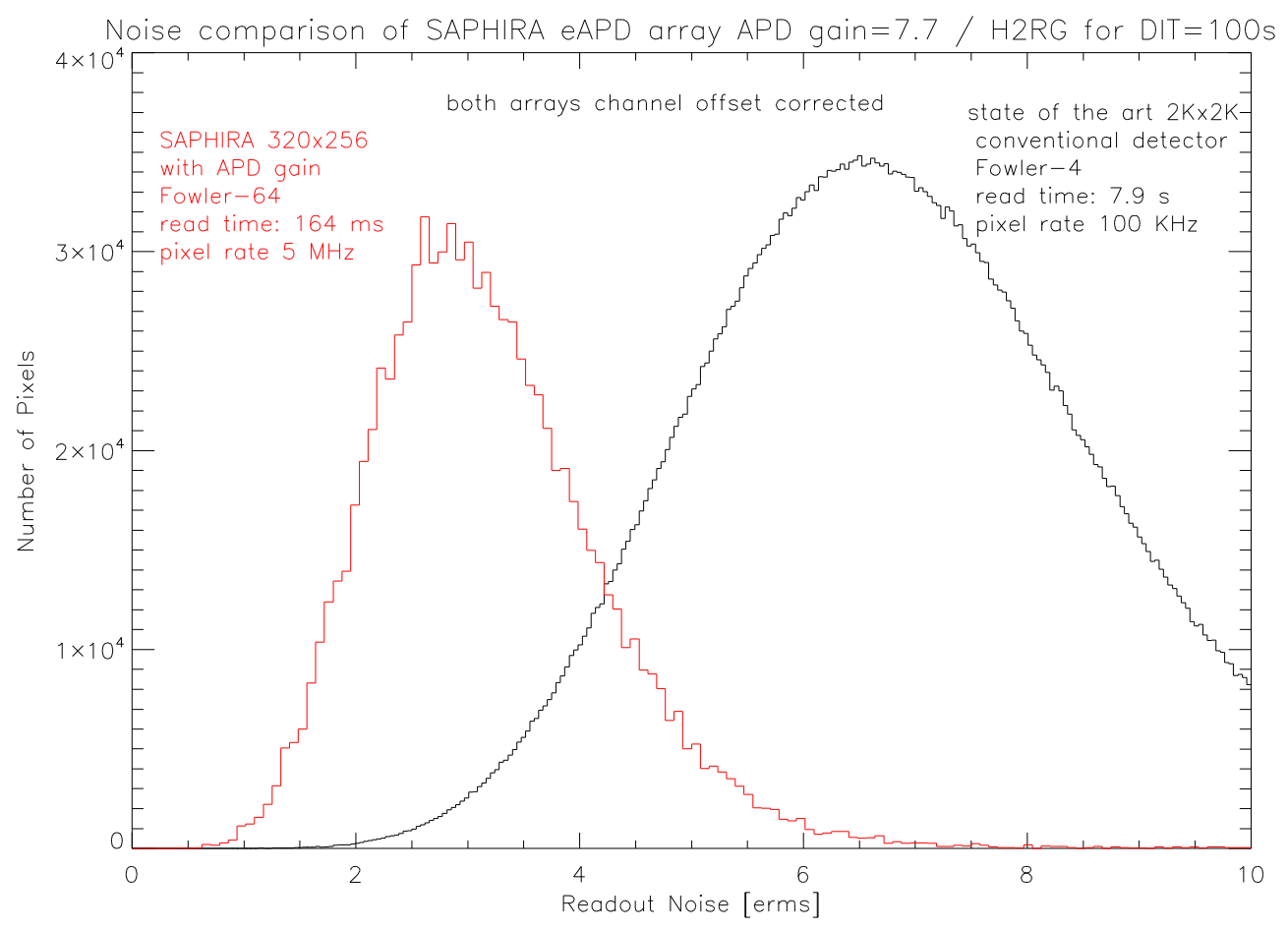

Figure 11 Noise comparison of SAPHIRA array with conventional state of the art $2 \mathrm{~K} \times 2 \mathrm{~K}$ array for long exposures with an integration time of 100s. Both arrays have been channel offset corrected. For fair comparison ratio of readout times is ratio of array formats. Red histogram: SAPHIRA array: noise 2.8 erms. Black histogram: conventional state of the art CMOS array: noise 6.5 erms. 


\section{CONCLUSIONS}

For control loops in the near infrared eAPD arrays are the devices of choice with unsurpassed performance as demonstrated by GRAVITY. The pixel performance of the current SAPHIRA arrays demonstrate that eAPD technology will also permit to make the next step in sensitivity for large science focal planes offering subelectron noise also for long integration times.

\section{REFERENCES}

[1] Gert Finger, Leander Mehrgan, Derek Ives, Siegfried Eschbaumer, Ian Baker, Reinhold Dorn, Jörg Stegmeier, Manfred Meyer, "Development of high-speed, low-noise NIR HgCdTe avalanche photodiode arrays for adaptive optics and interferometry" Proc. SPIE. 7742, High Energy, Optical, and Infrared Detectors for Astronomy IV, (2010).

[2] Gert Finger, Ian Baker, Domingo Alvarez, Leander Mehrgan, Jörg Stegmeier, Christophe Dupuy, Manfred Meyer, Harald Weller, Derek Ives, "Sub-electron read noise and millisecond full-frame readout with the near infrared eAPD array SAPHIRA” Proc. SPIE. 9909, Adaptive Optics Systems V

[3] Eisenhauer F. et al. "First light for GRAVITY", Proc. SPIE 9907, to be published, (2016).

[4] GRAVITY Collaboration (arXiv:1705.02345; A\&A 2017, in press)

[5] Hall D.N.B., "Towards the next generation of L-APD MOVPE HgCdTe arrays beyond the SAPHIRA $320 \mathrm{x}$ 256", Proc. SPIE 9915, (2016).

[6] Donald N. Hall, Ian Baker, Sean Goebel, Shane Jacobson, Charles Lockhart, Eric Warmbier, Dani Atkinson, "Next-generation performance of SAPHIRA HgCdTe APDs", Proc. SPIE. 9915, High Energy, Optical, and Infrared Detectors for Astronomy VII, (2016). 